\title{
Objetos de aprendizagem como coadjuvantes do processo de ensino- aprendizagem de Fisiologia humana
}

\section{Learning objects as coadjuvants in the human physiology teaching-learning process}

\author{
Marcus Vinícius Lara ${ }^{1}$, Sidnei Borges², Marcos Welter ${ }^{1}$, Pâmela Billig Mello-Carpes ${ }^{3 *}$ \\ *e-mail:panmello@hotmail.com \\ ${ }^{1}$ Graduando em Fisioterapia, Universidade Federal do Pampa - Unipampa. \\ ${ }^{2}$ Graduando em Farmácia, Universidade Federal do Pampa - Unipampa. \\ ${ }^{3}$ Laboratório de Estresse, Memória e Comportamento, Universidade Federal do Pampa - Unipampa. \\ Agência de fomento: CAPES
}

\begin{abstract}
Resumo
O uso de Tecnologias de Informação e Comunicação (TICs) no ambiente acadêmico na área biomédica tem ganhado muita importância, seja pela sua capacidade de complementar o entendimento da disciplina obtido em sala de aula, seja pela facilidade de acesso, ou por tornar mais prazeroso o processo de aprendizagem, uma vez que estas ferramentas estão presentes no cotidiano dos acadêmicos e utilizam uma linguagem simples. Considerando o exposto, este estudo objetiva relatar a experiência de construção de objetos de aprendizagem em fisiologia humana como uma ferramenta de facilitação do aprendizado, bem como discutir o impacto desta metodologia de ensino.
\end{abstract}

Palavras-chave: Tecnologia da Informação e Comunicação. Experimentação. Fisiologia. Recursos Audiovisuais.

\begin{abstract}
The use of Information and Communication Technologies (ICTs) in the academic environment of biomedical area has gained much importance, both for their ability to complement the understanding of the subject obtained in the classroom, is the ease of access, or makes more pleasure the learning process, since these tools are present in everyday of the students and use a simple language. Considering that, this study aims to report the experience of building learning objects in human physiology as a tool for learning facilitation, and discuss the impact of this teaching methodology
\end{abstract}

Keywords: Information and Communication Technologies. Experimentation. Physiology. Audiovisuals Resources. 
Inovações educacionais: Objetos de aprendizagem como coadjuvantes do processo de ensinoaprendizagem de Fisiologia humana

\section{Ficha da atividade desenvolvida}

Título: Objetos de aprendizagem como coadjuvantes do processo de ensinoaprendizagem de Fisiologia humana

Público alvo: Acadêmicos de graduação de cursos das áreas de ciências biológicas e da saúde.

Disciplinas relacionadas: Fisiologia Humana, Fisiologia Geral, Biologia, Biofísica, Bioquímica.

Objetivos educacionais: Utilizar a metodologia de construção de objetos de aprendizagem para dinamizar o trabalho dos conteúdos de disciplinas da área biomédicas que envolvam atividades práticas.

Justificativa de uso: Utilizar recursos de tecnologias da informação e comunicação, aos quais os alunos estão habituados no seu dia-a-dia, para facilitar o processo de ensinoaprendizagem de disciplinas que envolvem conteúdos compatíveis com experimentação e/ou aulas práticas.

Conteúdos trabalhados: Fisiologia celular, fisiologia da contração muscular, fisiologia sensorial, disfunções cerebelares, funções cognitivas, fisiologia do sangue e tecido imunológico, funções cardiovasculares, regulação da pressão arterial, fisiologia respiratória, processos de digestão, ação enzimática, função renal.

Link de acesso: http://oafisiologia.blogspot.com.br 
Inovações educacionais: Objetos de aprendizagem como coadjuvantes do processo de ensinoaprendizagem de Fisiologia humana

\section{Introdução}

Historicamente, embora tenham ocorrido algumas mudanças no processo de formação dos profissionais da área biológica e da saúde, ainda se costuma separar os objetos de seu contexto, as disciplinas umas das outras, o que faz com que o aluno tenha dificuldade de relacionar um conteúdo ao outro [1]. Costuma-se considerar apenas a dimensão de que o professor é aquele que ensina determinado conteúdo, em determinada área específica, deixando de considerar as dimensões pessoais, políticas, sociais, culturais, éticas do professor, de sua ação propriamente dita e de seus alunos [2].

Neste sentido, o ensino na área biomédica tem sido um desafio, principalmente quando a finalidade é a possibilidade de garantir uma aprendizagem efetiva e transformadora de atitudes e hábitos de vida [3]. Todavia, observa-se que nas últimas três décadas houve mudanças e agora vivemos em um mundo altamente tecnológico em que o acesso à informação é mais rápido e mais fácil [4]. Neste sentido, a busca por metodologias de ensino que promovam uma facilitação no processo de ensinoaprendizagem deve ser uma constante tarefa docente, considerando que desta maneira 0 conteúdo será disponibilizado com maior quantidade de recursos [5].

Assim, devemos considerar que a educação está, cada vez mais, inserida em um processo de globalização. Neste contexto, no qual as novas tecnologias ultrapassam os limites do corpo físico, transformando-se em extensões do pensamento e dos sentidos, vindo a potencializar as ações humanas [6], devemos considerar a utilização do computador como preponderante na configuração do conhecimento, diante de um acesso facilitado e no qual o aluno pode avançar de acordo com a sua velocidade [7].

Sem dúvida, hoje o computador merece destaque na sociedade, pois mais do que todas as outras inovações tecnológicas, ele provocou algumas mudanças no cotidiano e hábitos das pessoas, além disso, alterou a dimensão do acesso a conteúdos informativos, aliado ao prazer da utilização de seus recursos [8]. E, com os computadores, vieram as tecnologias da informação e comunicação (TICs). Na sua essência, as TICs desempenharam uma função interessante na prática pedagógica, auxiliando em diversos fatores por associar diferentes estímulos, facilitando a aprendizagem [9]. Com o advento das TICs houve uma revolução nos meios de comunicação [10]; diversas atividades humanas são hoje realizadas com mais rapidez e eficácia, distâncias físicas são superadas e a mídia eletrônica possibilita a informação circular em tempo real [8]. 
Inovações educacionais: Objetos de aprendizagem como coadjuvantes do processo de ensinoaprendizagem de Fisiologia humana

Dentre as diferentes ferramentas que as TICs englobam podemos destacar os Objetos de Aprendizagem (OA). OA são construções virtuais programadas que permitem designers, cores, movimentos, efeitos variados; são um novo tipo de instrução utilizando linguagens de computação [7]. Assim, OA podem ser definidos como recursos digitais que são usados, reutilizados e combinados com outros objetos para formar um ambiente de aprendizado rico e flexível [11]. Seu uso pode reduzir o tempo de desenvolvimento, diminuir a necessidade de instrutores especialistas e os custos associados com o desenvolvimento baseado em web [6].

Dentre as formas de OA mais amplamente utilizadas estão os vídeos didáticos [12]. Estes recursos audiovisuais solicitam constantemente a imaginação, que está intimamente interligada à afetividade, fazendo com que jovens e adultos respondam sensivelmente a linguagem utilizada [13]. Cabe salientar que o papel do professor ou do tutor é insubstituível nessas situações; são eles que ajudam a transpor barreiras e a suavizar ou mesmo resolver conflitos sócio-cognitivos [14].

O papel mediador do professor é um fator importante, pois ao se usar o vídeo em sala de aula, o OA pode se tornar para o estudante uma atividade de lazer, não sendo devidamente relacionado ao conteúdo, se não for corretamente orientado [14]. O vídeo também tem uma dimensão moderna e lúdica, pois é um meio de comunicação contemporâneo, novo e que integra várias linguagens e permite brincar com a realidade, isso sem considerar o fato de que é possível utilizá-lo aonde quer que seja necessário ou desejável [14].

Desta forma, verifica-se que o vídeo caracteriza uma poderosa ferramenta a qual o professor pode lançar mão; entretanto o professor deve corresponder à evolução das tecnologias para uma adequada utilização objetivando o feedback esperado [8]. Logo, a preparação para utilização do recurso se torna necessária, para que não haja uma utilização inadequada ou até mesmo falta de utilização desses recursos tecnológicos [15]. Contudo, observa-se que o uso desta tecnologia não tem alterado os modelos de ensinoaprendizagem, pois carrega velhas concepções pedagógicas, como, por exemplo, aquelas que não enfatizam a interação e participação discente [16]. Assim, apesar de todas as oportunidades oferecidas pela tecnologia, o uso do vídeo, por si só, não é eficiente sem uma boa estrutura pedagógica [10].

Existe um segredo para fazer com que o aluno participe do processo de ensinoaprendizagem de forma enérgica; para isso é necessário torná-lo autor, ou coautor, do 
Inovações educacionais: Objetos de aprendizagem como coadjuvantes do processo de ensinoaprendizagem de Fisiologia humana

processo de criação [17]. Algo que ele participou, ou que ele criou, toma uma dimensão muito diferente. Neste sentido os professores devem se apoderar de estratégias que podem cooperar no auxílio do processo de construção do conhecimento. A ação liberadora, provocada pela tecnologia do vídeo, quando colocada nas mãos dos alunos, permite a eles a experiência da pesquisa, do avaliar-se, do conhecer e conhecer-se [18].

É importante que o professor se aproprie das mídias para poder alcançar os seus alunos. A interação midiática enriquece os ambientes de aprendizagem. Torna-os mais atrativos, não só para os mais jovens, mas também para os adultos [14]. Por consequência, observamos que o uso de vídeo em sala de aula pode trazer grandes benefícios, visto que potencializa o aprendizado e amplifica o poder de compreensão dos alunos. Assim, é importante considerar o uso deste tipo de OA não só como um apoio ao ensino, mas também como uma fonte de produção de conhecimento e estímulo à pesquisa.

As ferramentas de web 2.0 buscam a participação discente e sua interação e, ainda, são importantes nas ciências, por sua integração em um corpo de conhecimento partilhado por cientistas [19]; a ciência em si é baseada na comunicação e divulgação dos resultados. Assim, a aplicabilidade das ferramentas da web 2.0 no contexto educacional possui quatro vertentes: pesquisa acadêmica, publicações, biblioteca e ensinoaprendizagem, as quais servem, respectivamente, para compartilhar resultados com a comunidade científica, para prover um meio mais rápido quando comparado coma as mídias tradicionais, para possibilitar novas formas de indexação e, no que se refere ao ensino-aprendizagem, o uso das ferramentas possibilita a realização de práticas educativas que valorizam a aprendizagem continuada e o desenvolvimento do espírito criativo e inovador dos alunos [20]. Neste sentido, o objetivo deste artigo é relatar as experiências da prática de construção de OA como ferramenta de apoio ao ensino da fisiologia humana, bem como discutir o impacto desta metodologia de ensino.

\section{Procedimentos e Recursos}

Este estudo foi realizado a partir da observação de vivências e práticas pedagógicas realizadas junto a alunos de graduação dos cursos de Enfermagem e Fisioterapia da Universidade Federal do Pampa, campus Uruguaiana - RS, no período de maio a novembro de 2011, totalizando a participação de aproximadamente 90 alunos. 
Inovações educacionais: Objetos de aprendizagem como coadjuvantes do processo de ensinoaprendizagem de Fisiologia humana

Para efetivação da proposta foram realizados os procedimentos detalhados a seguir.

A) Realização de experimentos práticos com orientação do docente e monitores da disciplina.

Esta primeira etapa foi realizada ao longo das disciplinas de Fisiologia Humana I e II, componentes curriculares obrigatórios dos cursos envolvidos. À medida que conteúdos teóricos foram trabalhados realizaram-se aulas com experimentos práticos, conforme já estabelecido no cronograma das disciplinas.

B) Filmagem do experimento.

A partir da realização das práticas, foi proposto aos alunos matriculados nas disciplinas de Fisiologia Humana que realizassem os experimentos novamente, com auxílio dos monitores das disciplinas, filmando-os.

C) Edição do vídeo do experimento.

Com o auxílio dos monitores, os vídeos produzidos pelos alunos foram editados, para se tornarem mais didáticos e com bom aspecto estético.

D) Construção de um roteiro de acompanhamento.

Após a finalização do vídeo aos acadêmicos construíram roteiros detalhados com instruções escritas de cada passo do experimento, a fim de complementar as informações do vídeo e facilitar a reprodução dos experimentos, se for o caso.

E) Disponibilização do vídeo na internet, em blog específico do projeto.

Os vídeos foram postados na internet, em um blog específico do projeto, denominado "Objetos de Aprendizagem em Fisiologia Humana" (http://oafisiologia.blogspot.com.br/), com livre acesso e espaço para comentários.

As temáticas dos vídeos construídos pelos alunos de graduação incluíram:

Homeostasia;

Fisiologia celular (transporte através da membrana celular);

Neurofisiologia (potenciação sináptica, movimentos reflexos);

Fisiologia da contração muscular (tipos de contração, velocidade e força da contração muscular esquelética);

Fisiologia sensorial (somestesia, visão, propriocepção, dor, audição, gustação, olfato e equilíbrio);

Fisiologia do sistema motor somático (disfunções cerebelares);

Funções cognitivas; 
Inovações educacionais: Objetos de aprendizagem como coadjuvantes do processo de ensinoaprendizagem de Fisiologia humana

Fisiologia do sangue e tecido imunológico;

Funções cardiovasculares (regulação da frequeência cardíaca e da pressão arterial);

Circulação arterial e venosa;

Fisiologia respiratória (mecânica ventilatória, volumes e capacidades pulmonares, espaço morto, tensão superficial);

Fisiologia digestória (processos de digestão, mastigação e deglutição, ação enzimática).

Ao longo das diversas etapas supracitadas (A-E) foi utilizada a observação participante, na medida em que professor e monitores envolvidos no projeto observaram, analisaram e anotaram informações acerca da participação, envolvimento e motivação dos alunos, informações estas utilizadas neste relato de experiência. Na observação participante os pesquisadores têm contato direto com os sujeitos, no seu contexto, sendo os próprios investigadores um instrumento de pesquisa [21,22]. Na sessão a seguir são apresentadas as percepções dos pesquisadores/observadores ao longo do estudo na perspectiva de discutir o impacto desta metodologia de ensino na prática pedagógica.

\section{Resultados e Discussão}

Percebemos que o uso de uma metodologia de ensino que envolve a construção de OA em uma disciplina da área biomédica, cujas práticas pedagógicas clássicas envolvem grande volume de conteúdo teórico, complementado por extensas leituras, promoveu maior envolvimento dos acadêmicos, proporcionando melhor compreensão dos fenômenos fisiológicos, maior interesse pela pesquisa científica e estimulando a busca voluntária por leituras científicas complementares, no sentido de qualificar a construção do grupo envolvido.

Nas diferentes etapas de construção dos OA observamos que os alunos leram mais, indo além dos livros didáticos e buscando atualizações por meio de artigos científicos. Esta busca, por sua vez, despertou em muitos deles o interesse pela ciência e pela pesquisa na área biológica. Ademais, percebemos que o envolvimento dos alunos com a metodologia utilizada foi além do simples cumprimento de uma tarefa dada pelo professor. Eles buscaram qualificar suas produções e mostraram-se motivados com os resultados, compartilhando suas criações e discutindo-as com os colegas. Na figura 01 é apresentada uma interface do blog do projeto, na qual estão disponíveis os OA 
Inovações educacionais: Objetos de aprendizagem como coadjuvantes do processo de ensinoaprendizagem de Fisiologia humana

construídos por uma das turmas participantes da atividade.

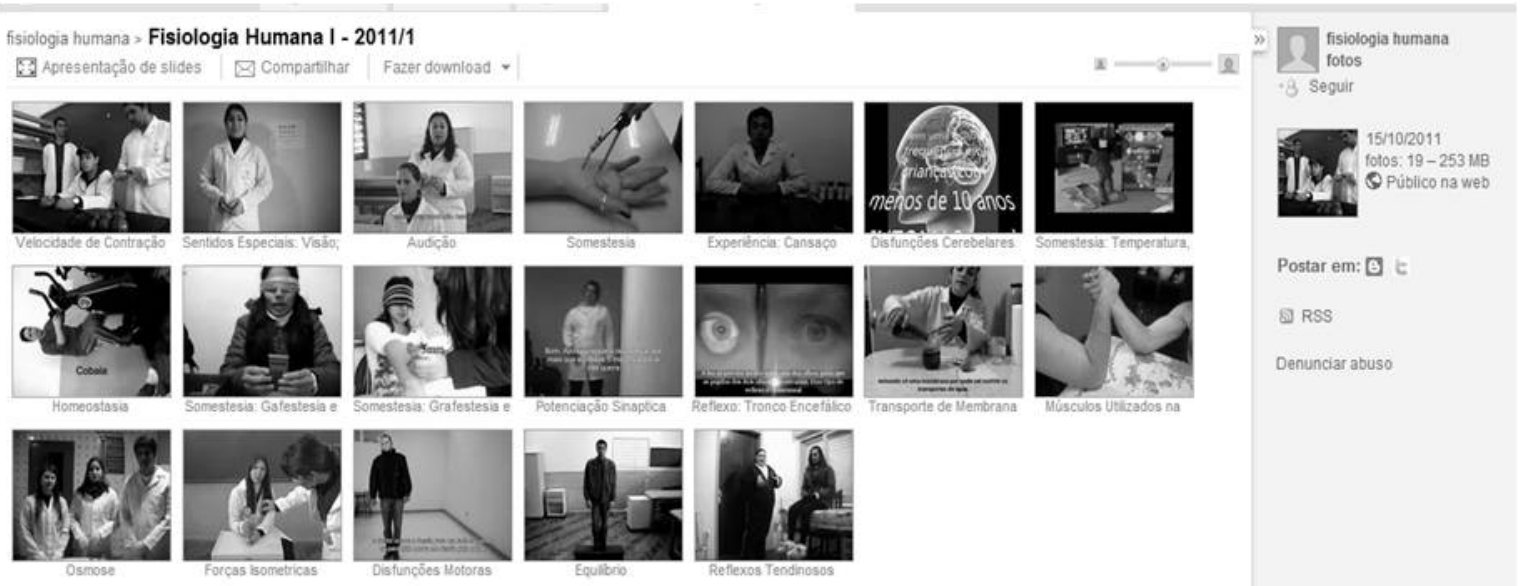

Figura 01. Interface do blog "Objetos de aprendizagem em Fisiologia Humana", na qual são disponibilizados os OA/vídeos criados por uma das turmas de Fisiologia Humana participantes do projeto.

Não só uma prática prazerosa para o aluno envolvido na construção do OA, nós observamos que o uso de TICs através das atividades propostas neste projeto contribuiu, também, para facilitar o processo de ensino-aprendizagem dos outros alunos matriculados nas disciplinas de Fisiologia Humana, ou relacionadas à essas, uma vez que os vídeos puderam ser livremente acessados e utilizados em outras disciplinas. Sabe-se que a quebra de ritmo provocada pela apresentação de um audiovisual em aula é saudável, pois altera a rotina da sala de aula [23], neste caso, como se trata de conteúdo de uma disciplina básica, o material pode ser utilizado posteriormente nos cursos de graduação, sendo assim também uma interessante a oportunidade de revisão deste conteúdo através do acesso aos OA construídos pelos próprios acadêmicos [24].

Conforme Pinheiro [25], qualquer tipo de TIC, desde ferramentas como bibliotecas digitais, repositórios virtuais, entre outros, apesar de terem sido criados com um propósito, podem exercer outras funções. A utilização das TICs no processo de ensinoaprendizagem em si, é recente, mas agora, vivemos em um ciberespaço [26], onde apresentamos a interface web de uma forma natural, de modo que o número de estudos nesta área ainda pode ser considerado pequeno, mas aos poucos começam a surgir novas publicações que demonstram seus benefícios e a boa aceitação pelos alunos. Nós percebemos que todos os alunos envolvidos demonstraram interesse na metodologia de trabalho proposta, sendo que a motivação percebida foi maior do que percebemos em grupos de alunos anteriores, aos quais propusemos o trabalho destes conteúdos utilizando metodologias de ensino tradicional. Nossos alunos reportaram como um dos 
Inovações educacionais: Objetos de aprendizagem como coadjuvantes do processo de ensinoaprendizagem de Fisiologia humana

principais fatores que os motivaram a estudar o protagonismo que tiveram na construção dos seus vídeos.

Martinho e Pombo (2012), em um estudo de caso, também relataram que a maioria dos alunos (92\%) considerou que tanto a projeção de imagens em PowerPoint, como o visionamento de vídeos educativos, bem como a pesquisa na Internet, influenciou positivamente a sua aprendizagem [9]; apontando, como razões principais, o fato destas estratégias melhorarem a atenção e aumentarem o entusiasmo de aprender. Contudo, este ambiente harmônico e didático, que é estimulado para estabelecer um vínculo entre os participantes dos cursos e os professores/monitores, e que parece simples, deve ser conduzido, indubitavelmente, com serenidade para alcançar resultados importantes.

Segundo Torezzan e Behar:

\begin{abstract}
A escola necessita estar preparada para interagir com essa realidade e adotar práticas pedagógicas que acompanhem e incentivem o desenvolvimento de uma postura autônoma e criativa por parte do aluno. Desse modo, os recursos digitais - imagens digitais, vídeos, animações, hipertextos, entre outros - vêm sendo cada vez mais aplicados em materiais educacionais, com o objetivo de contextualizar e possibilitar diferentes aprendizagens [27] (p.1).
\end{abstract}

Diante dos fatos contextualizados, cabe ressaltar também, que escolas/universidades e professores de diferentes níveis devem estar aptos a utilizar as TICs em sala de aula. As novas possibilidades de trabalho com OA proporcionam uma melhor organização aos alunos, como também elevam a qualidade das aulas ministradas e o seu envolvimento na realização dos mesmos. Deste modo, diminui o abismo entre a oportunidade dos alunos se tornarem ativos na construção do seu próprio conhecimento, de procurarem e explorarem áreas do seu interesse, e de construir um entendimento para os conhecimentos adquiridos. Alunos e professores encontram inúmeros recursos online que facilitam a tarefa de preparar as aulas e, fazer pesquisas, além de fontes de materiais atraentes para apresentação em sala de aula. Ainda, o processo de ensino-aprendizagem utilizando OA permite aos alunos desenvolver um pensamento crítico e reflexivo [28]. Este é outro aspecto que devemos relatar em relação às nossas observações. No momento em que os alunos passaram a ser orquestradores de experimentos e construtores e OA passaram a discutir criticamente os fenômenos observados e exigir explicações bem fundamentadas para os acontecimentos biológicos, o que é de extrema importância para o desenvolvimento da aprendizagem.

Cabe destacar, ainda, que, com o uso das TICs e dos OA o professor pode estar 
Inovações educacionais: Objetos de aprendizagem como coadjuvantes do processo de ensinoaprendizagem de Fisiologia humana

mais próximo do aluno, podendo adaptar a sua aula para o ritmo de cada um deles. $\mathrm{O}$ processo de ensino-aprendizagem pode ganhar dinamismo, inovação e comunicação inusitada [29]. A tarefa de melhorar nosso sistema educacional, dinâmico e complexo, exige atuação em múltiplas dimensões e decisões fundamentadas, seguras e criativas [30]. Projetos que visam implementar a diversidade da abordagem curricular tornam-se válidos a medida em que práticas novas de ensino tendem a tornar a disciplina mais atrativa para os acadêmicos, tornando-os agentes ativos na construção do conhecimento.

Assim, os OA são recursos digitais moduladores que podem ser usados para apoiar aprendizagem presencial e à distância [31], no entanto, para sua divulgação e maior alcance precisamos de recursos complementares. Diante disso, o blog foi utilizado nesta atividade para fins de compartilhamento dos OA entre os acadêmicos (na figura 02 é apresentada a página inicial do blog do projeto), permitindo que os alunos compartilhassem seus pensamentos e suas percepções de materiais, recursos e experiências de aprendizagem [32].

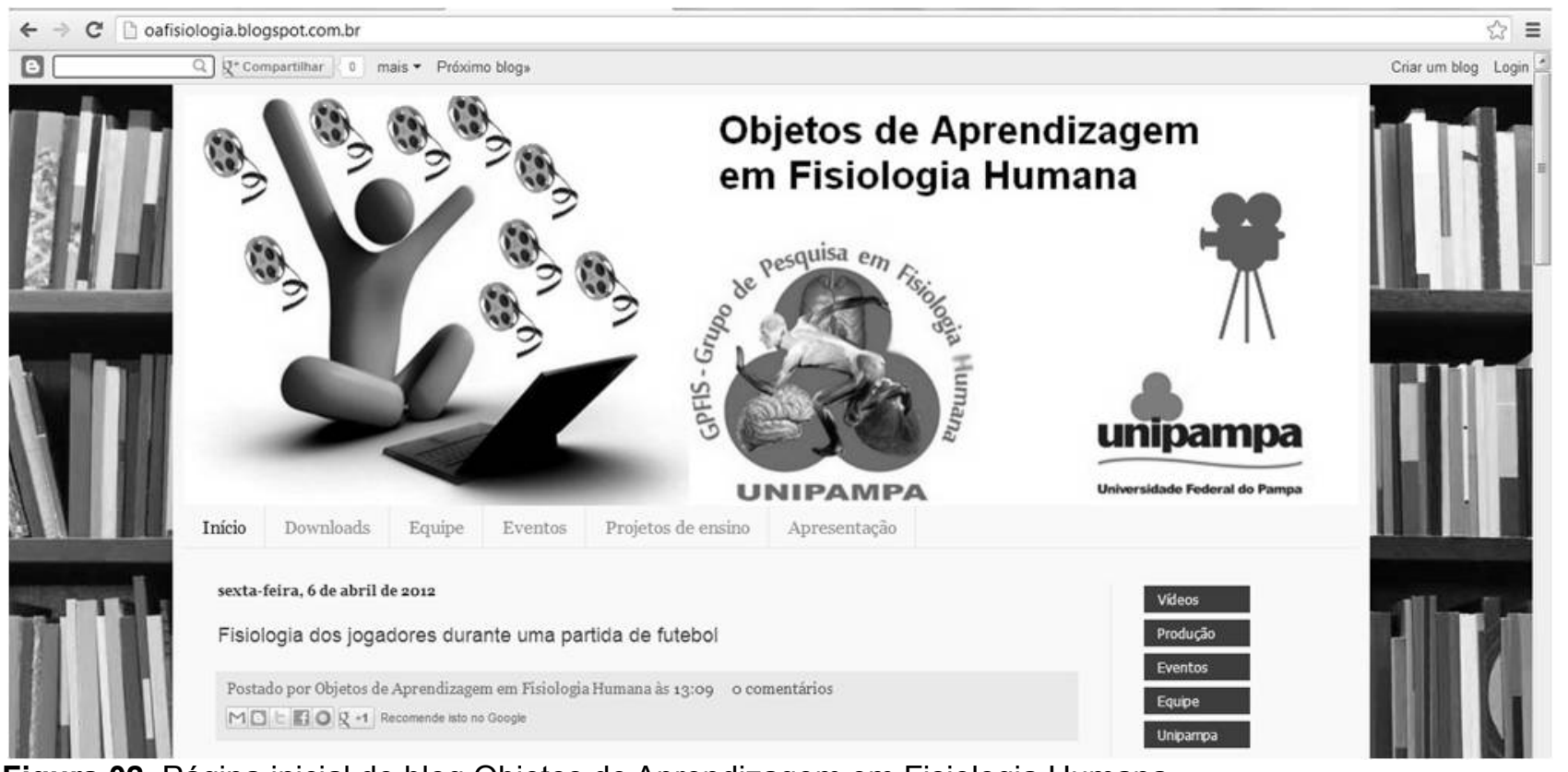

Figura 02. Página inicial do blog Objetos de Aprendizagem em Fisiologia Humana.

Encontramos várias aplicações para o blog, revelando que este tipo de ferramenta, devido à sua versatilidade e simplicidade para postar conteúdo de qualidade e, é um fator importante no processo de aprendizagem [27]. Nossos alunos acessaram o blog para conferir a postagem de seu vídeo e, também, para assistir os vídeos produzidos pelos colegas sobre outros conteúdos de fisiologia. Esta dinâmica permitiu, também, que eles avaliassem os OA produzidos pelos colegas, compartilhando dicas, oriundas de sua 
Inovações educacionais: Objetos de aprendizagem como coadjuvantes do processo de ensinoaprendizagem de Fisiologia humana

experiência prévia, e ideias, que poderiam ser incorporadas na sua construção.

No entanto, sabe-se que cada tipo de mídia requer um planejamento cuidadoso de seu uso, que vai além da disponibilidade dos equipamentos e da definição de seu uso em determinada aula, ou não [33]. Assim, o estudo destas novas metodologias, seu planejamento prévio e aplicação cuidadosa em sala de aula requerem tempo e dedicação docente e, na maioria das vezes, capacitação para seu uso [34], porém, seus resultados, especialmente na área biomédica, parecem promissores.

\section{Conclusão}

Neste trabalho podemos concluir que a utilização de uma metodologia de construção de OA constitui uma forma de facilitação do processo de ensino-aprendizagem da fisiologia humana. A observação da participação e envolvimento dos alunos permite predizer que esta prática não trouxe benefícios diretos somente ao ensino e aprendizagem da disciplina em si, mas também para as disciplinas posteriores e para o envolvimento dos alunos, monitores e docentes com o uso da TICs na educação. Além disso, as atividades aqui propostas prepararam os alunos para um melhor desempenho em outras disciplinas e permitiram a criação de OA que poderão ser utilizados a qualquer tempo e em qualquer local que permita acesso à internet; assim como estimularam o engajamento dos acadêmicos em atividades de ensino extraclasse, como a pesquisa científica.

Assim, os objetos de aprendizagem constituem uma forma de uso da tecnologia em sala de aula, que, com baixo custo, muita flexibilidade e alta capacidade de adaptação ao trabalho que o docente desenvolve, pode contribuir com o processo de ensinoaprendizagem, especialmente na área biomédica, porém o seu uso requer planejamento adequado.

\section{Referências}

[1] Morin E, Almeida MCX, Carvalho. Educação e complexidade: os sete saberes e outros ensaios. São Paulo: Cortez, 2002.

[2] Soares M. As pesquisas nas áreas específicas influenciando o curso de formação de professores. In: André M, Rosa DEG, Beillerot J, Santos, LLCP, Soares M, Miranda MG, et al. O papel da pesquisa na formação e na prática dos professores. $8^{\mathrm{a}}$ ed., Campinas: Papirus, 2001. 
Inovações educacionais: Objetos de aprendizagem como coadjuvantes do processo de ensinoaprendizagem de Fisiologia humana

[3] Duarte VS, Santos ML, Souza EJC, Borges GF. "Brincando com a fisiologia humana": Relato de uma extensão universitária. Revista Ciência em Extensão; 2012; 8(1): 98-106.

[4] Giddens A. Modernidade e Identidade. Rio de Janeiro: Jorge Zahar, 2002.

[5] Corradi MI, Silva SH, Scalabrin EE. Objetos virtuais para apoio ao processo ensinoaprendizagem do exame físico em enfermagem. Acta Paulista de Enfermagem; 2001; 24(8): 348-53.

[6] Antonio Junior W, Barros DMV. Objetos de aprendizagem virtuais: material didático para a educação básica. 12. Congresso Internacional de Educação a Distância [Internet]. 2005. [acesso em 20 de abril de 2012]. Disponível em:

http://www.abed.org.br/congresso2005/por/pdf/006tcc1.pdf.

[7] Cogo ALP, Pedro ENR, Silveira DT, Silva APSS, Alves RHK, Catalan VM. Development and use of digital educative objects in nursing teaching. Rev. Latino-Am. Enfermagem; 2007; 15(4): 699-701.

[8] Sá Filho CS, Machado EC. O computador como agente transformador da educação e o papel do objeto de aprendizagem. 1. Seminário Nacional ABED de Educação a Distância: "Habilidades e Talentos em EAD" [Internet]. 2003. [acesso em 20 de abril de 2012]. Disponível em: http://www.abed.org.br/seminario2003/texto11.htm.

[9] Martinho T, Pombo L. Potencialidades das tic no ensino das ciências naturais - um estudo de caso. Revista Electrónica de Enseñanza de las Ciencias [Internet]. 2009. [acesso em 02 de maio de 2012]. Disponível em: http://www.saum.uvigo.es/reec/volumenes/volumen8/ART8_Vol8_N2.pdf.

[10] Régis F. Tecnologias de comunicação, entretenimento e competências cognitivas na cibercultura. Revista Famecos; 2008; 1(37): 32-37.

[11] Alvarez AG, Sasso GTMD. Objetos virtuais de aprendizagem: contribuições para o processo de aprendizagem em saúde e enfermagem. Acta Paul Enferm; 2011; 24(5): 70711.

[12] Tarouco LMR, Rodrigues AP, Schimtt MAR. Integração do MOODLE com repositórios abertos. Perspect Ciênc Inf; 2013; 18(1): 66-85.

[13] Morán JM. O vídeo na sala de aula. Revista Comunicação e Educação; 1995; (2): 2735.

[14] Caetano SVN., Falkembach GAM. YOU TUBE: uma opção para uso do vídeo na EAD. Renote; 2007; 5(1): 1-10.

[15] Valle LRLD, Cruz DM. Reinventando a TV e o Vídeo na Escola: Uma experiência com a TV Escola e os professores da Rede Estadual de Ensino do Paraná. Athena: Revista Científica de Educação; 2003; 1(1): 9-11.

[16] Andrade A. Uso(s) das novas tecnologias em um programa de formação de professores: possibilidades, controle e apropriações. Dissertação de Mestrado [Educação] Universidade de São Paulo. 2007.

[17] Santoro F, et al. Autoria cooperativa de crianças em sistemas hipermídias: relato de uma experiência 1997. In: ED-MEDIA .1997, Calgary. Anais... Calgary, 1997. 
Inovações educacionais: Objetos de aprendizagem como coadjuvantes do processo de ensinoaprendizagem de Fisiologia humana

[18] Ferrés J. Vídeo e Educação. $2^{\mathrm{a}}$ ed. Porto Alegre: Artes Médicas. 1996.

[19] Cabo JV, Martín-Rodero H. Acceso Abierto: nuevos modelos de edición científica en entornos web 2.0. Salud colectiva; 2001; 7(1): 19-27.

[20] Anderson P. What is web 2.0? Ideas, technologies and implications for education. JISC Technology and Standards Watch [Internet]. 2007. [acesso em 20 de abril de 2012]; 2-64. Disponível em: http://www.jisc.ac.uk/media/documents/techwatch/tsw0701b.pdf.

[21] Bogdan R, Taylor S. Introduction to qualitative research methods: a phenomenological approach to the social sciences. $2^{\mathrm{a}}$ ed. New York: J. Wiley. 1975.

[22] Correia MCB. A observação participante enquanto técnica de investigação. Revista Pensar Enfermagem; 2009; 13(2): 30-6.

[23] Rosa PRS. O uso dos recursos audiovisuais e o ensino de ciências. Caderno Catarinense de Ensino de Física; 2000; 17(1): 33-49.

[24] Santa-Rosa JG, Struchiner M. Tecnologia educacional no contexto de ensino de Histologia: Pesquisa e desenvolvimento de um ambiente virtual de ensino e aprendizagem. Rev Bras Educ Med; 2011; 35(2): 289-98.

[25] Pinheiro LVR. Internet, ciência e sociedade: o que mudou para pesquisadores e cidadãos? Revista Com Ciência; 2012; (141): 1-2.

[26] Santaella L. Os espaços líquidos da cibermídia. Revista da Associação Nacional dos Programas de Pós-Graduação em Comunicação; 2005; (2): 1-13.

[27] Torrezzan CAW, Behar PA. Design Pedagógico de Materiais Educacionais Digitais. 5. Congresso Brasileiro de Educação Superior a Distância [Internet]. [acesso em 09 de maio de 2012]. Disponível em: http://200.169.53.89/download/CD\%20congressos/2008/V \%20ESUD/trabs/t38664.pdf.

[28] Del Moral ME, Villalustre L. Herramientas digitales para facilitar el "blended learning" y el desarrollo de competencias: Webquest y Weblog. In: Del Moral ME, Cernea DA, Martínez LV. Objetos de aprendizaje 2.0: Una nueva generación de contenidos en contextos conectivistas. Revista de Educacíon a Distancia; 2010; (25): 1-11.

[29] Mercado LPL, Silva AL, Gracindo HBR. Utilização didática de Objetos digitais de aprendizagem na educação online. Eccos Revista Científica; 2008; 10(1): 105-23.

[30] Valente JA, Freire FM, Rocha HV, D'Abreu JV, Baranauskas MCC, Martins MC, Prado MEBB. O computador na sociedade do conhecimento. Campinas: Editora Unicamp. 1999.

[31] Winner D. What makes a weblog a weblog? Weblog At Harvard Law School [Internet] 2003. [acesso em 01 de outubro de 2012]. Disponível em:

http://blogs.law.harvard.edu/whatMakesAWeblogAWeblog.

[32] Behar PA, Gaspar MI. Uma perspectiva curricular com base em objetos de aprendizagem. Virtual Educa Brasil [Internet] 2007. [acesso em 07 de maio de 2012]. Disponível em: http://ihm.ccadet.unam.mx/virtualeduca2007/pdf/37-PB.pdf.

[33] Kenski VM. Gestão e uso das mídias em projetos de educação à distância. Revista ECurriculum [Internet] 2005. [acesso em 07 de maio de 2012]. Disponível em: 
Inovações educacionais: Objetos de aprendizagem como coadjuvantes do processo de ensinoaprendizagem de Fisiologia humana

http://revistas.pucsp.br/index.php/curriculum/article/view/3099/2042.

[34] Anjos, LFR. A percepção de especialistas sobre as tecnologias de informação e comunicação no ensino de ciências. Trabalho de Conclusão de Curso [Ciências Naturais]. Universidade de Brasília. 2013.

\section{Agradecimentos}

Os autores agradecem ao Programa de Bolsas de Apoio ao Desenvolvimento Acadêmicos da Universidade Federal do Pampa (PBDA/UNIPAMPA), pelo apoio na forma de bolsas de iniciação ao ensino e à extensão, fornecidas aos alunos envolvidos na execução deste projeto. Os autores também agradecem aos alunos dos cursos de Fisioterapia e Enfermagem da UNIPAMPA, que abraçaram a ideia de construção de OA junto às disciplinas de Fisiologia Humana, envolvendo-se ativamente neste processo. 